\title{
Logic Circuit Equivalence Checking Using Haar Spectral Coefficients and Partial BDDs
}

\author{
M. A. THORNTON ${ }^{\mathrm{a}, *}$, R. DRECHSLER ${ }^{\mathrm{b}}$ and W. GÜNTHER ${ }^{\mathrm{c}}$

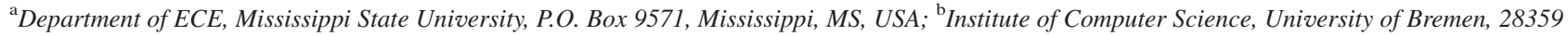 \\ Bremen, Germany \\ ${ }^{\mathrm{c}}$ Albert-Ludwigs-University, Freiburg, Germany
}

(Received 20 January 2000; In final form 4 October 2000)

\begin{abstract}
A probabilistic equivalence checking method is developed based on the use of partial Haar Spectral Diagrams (HSDs). Partial HSDs are defined and used to represent a subset of Haar spectral coefficients for two Boolean functions. The resulting coefficients are then used to compute and to iteratively refine the probability that two functions are equivalent. This problem has applications in both logic synthesis and verification. The method described here can be useful for the case where two candidate functions require extreme amounts of memory for a complete BDD representation. Experimental results are provided to validate the effectiveness of this approach.
\end{abstract}

Keywords: Verification; BDD; Spectral methods; Haar transform; Equivalence checking

\section{INTRODUCTION}

The equivalence checking problem for two Boolean functions of $n$ variables, $f(X)$ and $g(Y)$, is addressed in this work. Here, we assume that the correspondence between the vectors of variables, $X$ and $Y$ is known. Although this problem is easily solved when $\bar{f}$ and $g$ can be completely represented in BDD form, problems can arise for some functions whose corresponding BDD representations are too large. Thus, we have motivation to formulate a technique for equivalence checking based on partial representations of $f$ and $g$. The incorporation of the Haar spectral coefficients in our approach allows for further information about the two candidate functions to be exploited.

This problem has applications in logic synthesis in terms of the library binding stage where a technologically independent sub-function, $f$, must be "mapped" to a technologically dependent "library cell" represented functionally by $g$ such that $f(\underline{X})=g(\underline{Y})$ [13]. Typically, a subset of $g_{i}$ cells satisfy this equivalence and the logic synthesis system chooses a specific $g_{i}$ based on some optimization constraint such as area minimization, shape factor, speed, etc. Determining the appropriate set of "library cells", $\left\{g_{i}\right\}$, can be accomplished via the application of an equivalence checking technique.

The equivalence checking function is also of concern in verification systems where two representations of a function are compared [3,5,14,15,17]. Two abstractions of a circuit resulting from different optimization phases of a logic synthesis system (e.g. $f(X)$ and $g(Y)$ ) may need to be checked to determine if $f(\underline{X}) \equiv g(\underline{Y})$. This is applicable for methods that express state machines as BDDs as well as for the verification of purely combinational logic.

In many cases, this problem can be easily solved by building an Ordered Binary Decision Diagram (OBDD) $[1,6]$ representing $f$ and $g$ according to a common variable order. When this is possible, the determination of equivalence is accomplished by simply comparing two pointer values. However, some classes of functions result in OBDDs with an exponential number of vertices regardless of the variable order [2,4].

The technique described here allows for the equivalence checking problem to be formulated in terms of a subset of Haar spectral coefficients [10,11]. Given a set of Haar spectral coefficients, we examine the probability that $f(\underline{X})=g(\underline{Y})$. This allows the equivalence checking problem to be iteratively refined in terms of possible error by accounting for the existence of more matching coefficients. Thus, techniques that provide subsets of Haar spectral coefficients $[8,9,18]$ for representations of $f$ and $g$ can be used for non-tautology checking. A similar approach using an arithmetic transform and a decision diagram structure known as an Interleaved BDD (IBDD) has also been proposed [12]. The technique described here

*Corresponding author. Tel.: +1-662-325-3629. Fax: + 1-662-325-2298. E-mail: m.thornton@computer.org 
differs due to the fact that we utilize partial HSDs versus IBDDs allowing us to make use of the multi-resolution, modified Haar wavelet transform $[10,11]$ rather than the arithmetic transform. This allows for the advantage of partially representing the functions under consideration and to obtain the Haar spectral coefficients directly from a traversal of the HSD without performing additional spectral computations. Furthermore, the multi-resolution nature of the Haar transform offers advantages in the probability calculations since higher ordered coefficients can represent disjoint portions of the function of interest.

In this approach, we adapt the method reported in Ref. [9] that allows the Haar spectral coefficients to be represented as a HSD with the concept of the partial BDD as given in [16]. This allows for a partial function representation to be used for quickly computing subsets of Haar spectral coefficients avoiding problems that may arise for functions that result in very large BDDs when represented in their fully specified form. Once the subsets of Haar spectral coefficients are found to be equivalent for two candidate functions, $f$ and $g$, we compute the probability that $f$ and $g$ are equivalent. If any two same-ordered Haar spectral coefficients are found that have different values, we can declare that $f \neq g$ and halt the process.

A discussion of the background of partial BDDs and HSDs is reviewed followed by a section on the mathematical basis of our technique. The mathematical basis includes a review of relevant aspects of the Haar transform and contains the derivations for the probability computations. Next, we present a simple example and the results of some preliminary experiments that indicate the effectiveness of using matching Haar coefficients for statistical verification. Finally, a section containing conclusions is given.

\section{BINARY DECISION DIAGRAMS}

Boolean variables can assume values from $\mathbf{B}=\{0,1\}$. In the following, we consider Boolean functions $f: \mathbf{B}^{n} \rightarrow \mathbf{B}^{m}$ over the variables specified by the vector $(\underline{X})=\left(x_{1}, \ldots, x_{n}\right)$. As is well known, each Boolean function $f: \mathbf{B}^{n} \rightarrow \mathbf{B}$ can be represented by a Binary Decision Diagram (BDD) [1], which is directed acyclic graph where a Shannon decomposition

$$
f=\bar{x}_{i} f_{x_{i}=0}+x_{i} f_{x_{i}=1} \quad(1 \geq i \geq n)
$$

is carried out in each node.

A BDD is called ordered if each variable is encountered at most once on each path from the root to a terminal node and if the variables are encountered in the same order on all such paths. A BDD is called reduced if it does not contain multiple isomorphic sub-graphs or any instances of both edges from a single vertex pointing to the same node. Reduced and ordered BDDs are unique since each distinct Boolean function and a given variable ordering result in a canonical representation.

BDDs are defined analogously for multi-output functions $f: \mathbf{B}^{n} \rightarrow \mathbf{B}^{m}$ as for the case of single-output functions: A BDD $G_{j}$ for each component function $f_{j}(1 \geq$ $j \geq m$ ) is used for the shared BDD representation $G$ for $f$. The order of the variables is fixed over all $G_{j}$ s.

For functions represented by reduced, ordered BDDs, efficient manipulation algorithms may be formulated [1]. In the following discussion, only reduced, ordered BDDs are considered and for briefness these graphs are referred to as BDDs.

\section{INCOMPLETE CONSTRUCTION}

As long as symbolic simulation can be carried out completely, the verification process succeeds. But problems arise if BDDs do not fit in the main memory of a computer. This might be due to several reasons.

The first (and simplest) reason is that a "bad" variable ordering has been chosen. In the past, several techniques have been proposed for BDD minimization (for an overview see Ref. [6]). Furthermore, the ordering in which the operands are combined is very important, as can be seen by the following simple example:

Example 1 Let $\mathscr{F}$ be an AND gate with three inputs $f, g$ and $h$ that occur during symbolic simulation of a circuit. BDD packages based on recursive synthesis have to compute:

$$
(f \cdot g) \cdot h, f \cdot(g \cdot h) \quad \text { or } \quad(f \cdot h) \cdot g
$$

The order in which the calculation is performed largely influences the number of nodes that are needed during the computation, e.g. if $f \cdot g$ is computed first, but $h=0$. In this case, the result $f \cdot g$ (which might be large) is computed first even though the results of the AND gate is 0 .

Some first steps for finding good orderings involve traversing gates in circuit representations as described in Ref. [7]. However, there also exist functions for which the corresponding BDD size becomes exponential (independent of the variable ordering). The most popular example is the multiplier [2].

In Ref. [16], an approach based on partial information has been proposed: if the BDD size becomes too large some parts can be projected to a new "terminal" node, called $U$ for undefined. The drawback of this method is that the complete functionality of the represented circuit is no longer present and complete verification is not possible.

Fortunately, the resulting partial DD gives enough information to compute at least some Haar coefficients. This fact will allow us to formulate the equivalence checking technique based on matching subsets of Haar coefficients using only the partial BDDs.

To give a better understanding of partial BDDs, including the value $U$, we consider the function from Ref. [16] given by the table in Fig. 1. As can be seen, the BDD for this function requires 6 non-terminal nodes (in the following, we fix the variable ordering). We assume that the memory of the BDD packages is limited to four non-terminals nodes. Thus, complete construction is not 

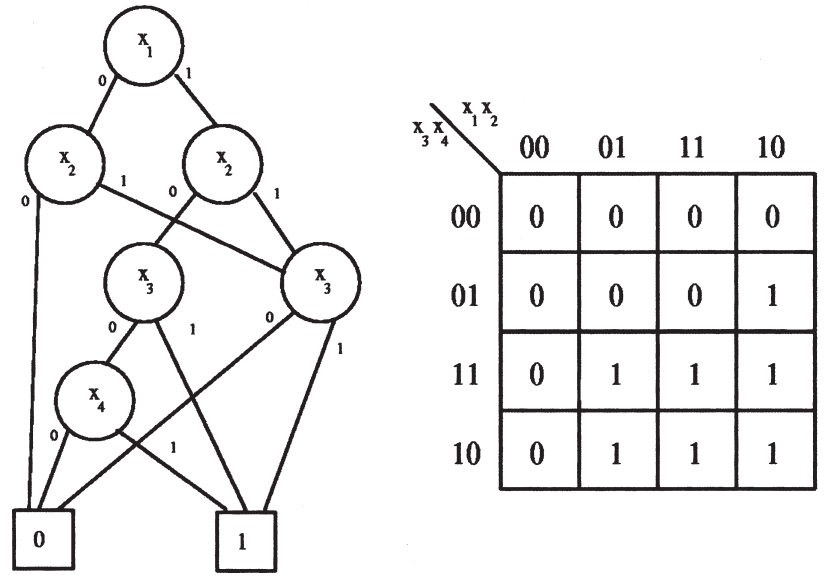

FIGURE 1 Complete BDD.

possible. However, if two runs are made using partial information and the rest is projected to an undefined value, $U$ (see Figs. 2 and 3), we see that the complete function can be obtained using two partial BDDs.

\section{HAAR SPECTRAL DIAGRAMS}

In Ref. [9], a directed graph referred to as a Haar Spectral Diagram (HSD) is defined that represents the Haar spectrum of a Boolean function. HSDs are isomorphic to BDDs (with the exception that all BDD terminal vertices are "mapped" to a common HSD terminal vertex). This allows the BDD representation of a function to double as a representation of the Haar spectrum with extra memory storage required only in the form of an additional edge-attribute value. The additional storage is needed because all 1-edges in the HSD have a Haar spectral coefficient as an attribute.

The enabling observation for defining the HSD is that the Haar transformation matrix can be expressed in terms of Kronecker products in natural order. The $n$-dimensional transformation matrix that produces the coefficients in
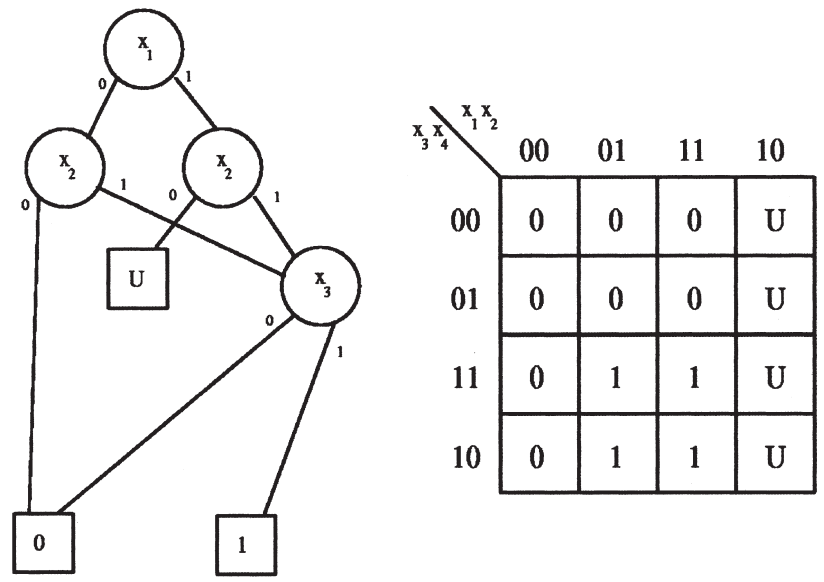

FIGURE 2 First incomplete BDD.

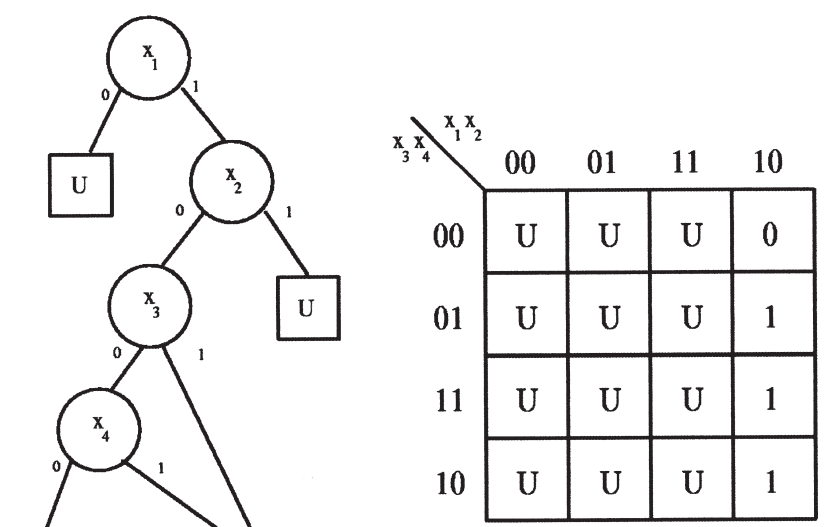

FIGURE 3 Second incomplete BDD.

natural order, $T^{n}$, can be represented as a sum of matrices denoted as $A^{n}$ and $D^{n}$ as given in Eq. (1).

$$
T^{n}=A^{n}+D^{n}
$$

$A^{n}$ can now be defined by the Kronecker product relation (denoted by the $\otimes$ operator) as:

$$
A^{n}\left[\begin{array}{ll}
1 & 0 \\
0 & 1
\end{array}\right] \otimes A^{n-1}+\left[\begin{array}{rr}
0 & 0 \\
1 & -1
\end{array}\right] \otimes D^{n-1}
$$

The initial cases are $A^{0}=0$ and $D^{0}=1$. It is observed that the first row of $A^{n}$ is all zeros and only the first row of $D^{n}$ is non-zero, thus the spectral vector due to $T^{n}$ can be represented by the two vectors due to $A^{n}$ and $D^{n}$ separately. By using this observation and viewing a nonterminal node of a BDD as pointing to two disjoint subfunctions, we can represent the spectrum of the subfunctions (the subfunctions spectra are actually scaled by a constant in this case) as two different portions of the entire vector due to $T^{n}$. Figure 4 is similar to the diagram originally appearing in Ref. [9] and illustrates this relationship.

Using these observations, it is possible to represent the Haar spectrum of a function by annotating all 1-edges of the graph (and the pointer to the initial node) with Haar spectral coefficients.

As an example, consider the Boolean function, $f=$ $x_{1} x_{2}+\bar{x}_{3}$. Equation (3) gives the Haar spectrum for this

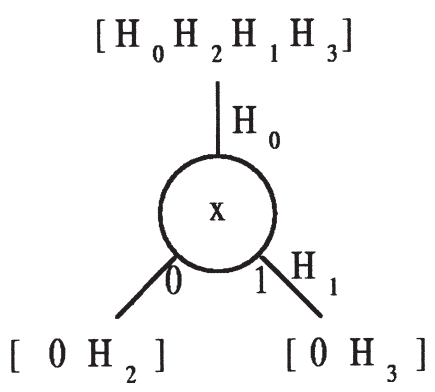

FIGURE 4 Non-terminal in HSD. 


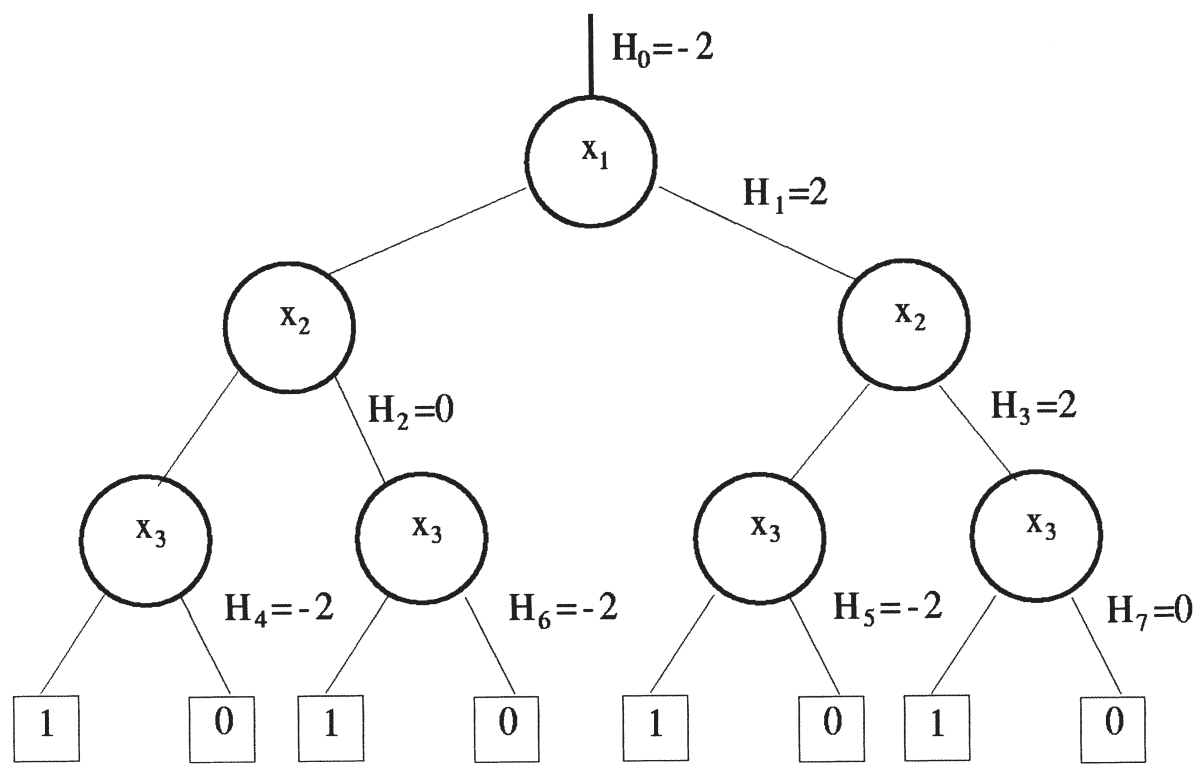

FIGURE 5 Shannon decision tree with Haar coefficient 1-edge annotations.

function. If the $T^{3}$ matrix were used, the resulting spectral vector would become $\left[H_{0}, H_{4}, H_{2}, H_{5}, H_{1}, H_{6}, H_{3}, H_{7}\right]=$ $[-2,-2,0,-2,2,-2,2,0]$. These coefficients can be used as annotation values on the Shannon decision tree representing the example function as shown in Fig. 5. As is well known, the corresponding BDD can be formed by removing all isomorphic subgraphs and redundant nodes from the decision tree representation. If these reductions are carried out and the edge annotations are retained, the BDD/HSD results as shown in Fig. 6.

$$
\begin{aligned}
& {\left[\begin{array}{rrrrrrrr}
1 & 1 & 1 & 1 & 1 & 1 & 1 & 1 \\
1 & 1 & 1 & 1 & -1 & -1 & -1 & -1 \\
1 & 1 & -1 & -1 & 0 & 0 & 0 & 0 \\
0 & 0 & 0 & 0 & 1 & 1 & -1 & -1 \\
1 & -1 & 0 & 0 & 0 & 0 & 0 & 0 \\
0 & 0 & 1 & -1 & 0 & 0 & 0 & 0 \\
0 & 0 & 0 & 0 & 1 & -1 & 0 & 0 \\
0 & 0 & 0 & 0 & 0 & 0 & 1 & -1
\end{array}\right]} \\
& \times\left[\begin{array}{r}
-1 \\
1 \\
-1 \\
1 \\
-1 \\
1 \\
-1 \\
-1
\end{array}\right]=\left[\begin{array}{r}
-2 \\
2 \\
0 \\
2 \\
-2 \\
-2 \\
-2 \\
0
\end{array}\right]
\end{aligned}
$$

The entire spectrum can be recovered through traversals of the HSD and several properties arise due to the BDD reduction rules. As an example, any 1-edge that is annotated by a negative value must point to either another non-terminal node or the logic-0 terminal. Likewise, a positive valued 1-edge attribute implies the reverse of this rule. Furthermore, if a 1-edge attribute is 0 -valued, it cannot point to a terminal node since it would be removed by the deletion rule. With knowledge of these properties and a given HSD, the entire spectrum can easily be reconstructed.

Using the example HSD in Fig. 6, it is immediately apparent that $H_{0}=-2, H_{1}=2$ and $H_{3}=2$ since these values are explicit 1-edge attributes. It is also inferred that $H_{4}=H_{5}=-2$ since all traversals from the $x_{1} 0$-edge do not encounter any $x_{2}$ node. $H_{6}=-2$ by the attribute value on the 1-edge from the $x_{3}$ node also since this edge is

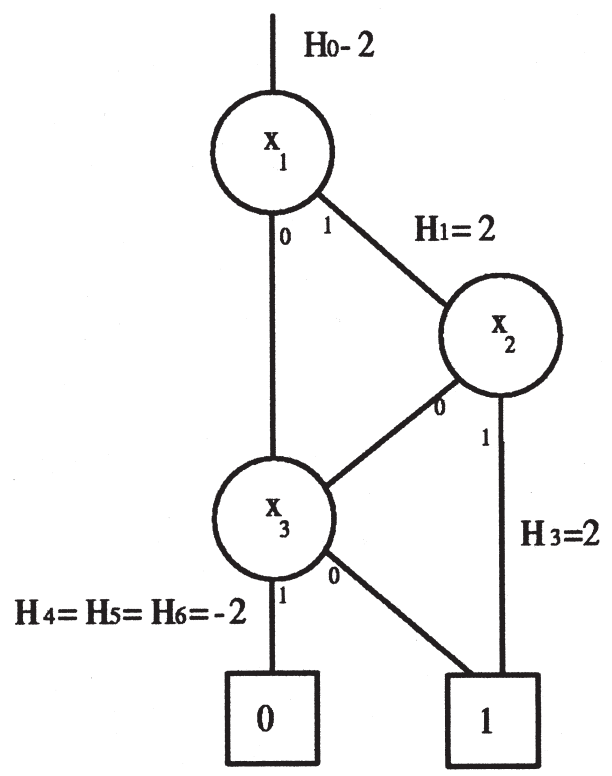

FIGURE 6 Example HSD. 
traversed for the path where $x_{1}=1, x_{2}=0$ and $x_{3}=1$. Also, $H_{2}=0$, since the leftmost $x_{2}$ node was removed from the Shannon decision tree due to its redundancy. Likewise, $H_{7}=0$ since the rightmost $x_{3}$ node was removed from the Shannon tree due to its redundancy.

\section{MATHEMATICAL BASIS AND DERIVATION}

In this section, the notation used throughout the remainder of the paper is defined and relations between probabilistic events and Haar spectral coefficients are derived.

\section{Notation}

The following notation is used:

- $f\left(x_{1}, x_{2}, \ldots, x_{n}\right)$ represents a fully specified Boolean function of $n$ variables that may also be represented by the vector, $\underline{X}=\left(x_{1}, x_{2}, \ldots, x_{n}\right)$.

- $H^{T}$ represents the transpose of the modified Haar spectral coefficient vector representing some function, $f(X)$.

- $\overline{H_{i}}(f)$ represents the individual $i$-th Haar spectral coefficient of the Boolean function, $f(X)$, where $\underline{H}^{T}=\left(H_{0}, H_{1}, \ldots, H_{2^{n}-1}\right) . H_{i}$ is also represented as $H_{s}^{o}$ in some of the literature where $o$ is the order of the spectral coefficient and $s$ is the $s$-th Haar function [11].

- $P[A]$ is the discrete probability that some event, $A$, occurs.

- $P[f]$ is the output probability of a Boolean function, $f$, which is the likelihood that $f=1$ given the distribution of the dependent variables in $X$.

- $S_{i}$ is the event that $H_{i}(f)=H_{i}(g)$, that is, the $i$-th Haar spectral coefficients of $f$ and $g$ are equal in value.

- $E$ is the event that $f(\underline{X})=g(\underline{Y})$, that is, the functions $f$ and $g$ are functionally equivalent.

\section{Haar Spectrum}

This section will summarize the ideas about how output probabilities can be used to compute the modified Haar spectral coefficients directly as given in Ref. [18]. The idea was developed by making observations about the structure of the transformation matrix.

Each transformation matrix row consists of the integer elements $-1,+1$ and 0 . An integer -1 represents the Boolean 1 constant, an integer +1 represents the Boolean 0 constant, and an integer 0 indicates the absence of a Boolean constant. Each row represents a particular modified Haar function, $f_{c}$, dependent upon $n$ or fewer variables where $n$ is the number of variables of $f$, the function to be transformed.

Figure 7 contains the modified Haar transformation matrix for any function of $n=3$ variables. It is noted that higher ordered coefficients are computed from matrix row functions with a decreasing range space dimension. In

\begin{tabular}{|c|c|c|c|c|c|c|c|c|}
\hline$f[$ & 1 & 1 & 1 & 1 & 1 & 1 & 1 & 1 \\
\hline$x_{1}$ & 1 & 1 & 1 & 1 & -1 & -1 & -1 & -1 \\
\hline$x_{2} \cdot f_{\bar{x}_{1}}$ & 1 & 1 & -1 & -1 & 0 & 0 & 0 & 0 \\
\hline$x_{2} \cdot f_{x_{1}}$ & 0 & 0 & 0 & 0 & 1 & 1 & -1 & -1 \\
\hline$x_{3} \cdot f_{\bar{x}_{1} \bar{x}_{2}}$ & $1-$ & -1 & 0 & 0 & 0 & 0 & 0 & 0 \\
\hline$x_{3} \cdot f_{\bar{x}_{1} x_{2}}$ & 0 & 0 & 1 & -1 & 0 & 0 & 0 & 0 \\
\hline$x_{3} \cdot f_{x_{1} \bar{x}_{2}}$ & 0 & 0 & 0 & 0 & 1 & -1 & 0 & 0 \\
\hline$x_{3} \cdot f_{x_{1} x_{2}}$ & & 0 & 0 & 0 & 0 & 0 & 1 & -1 \\
\hline
\end{tabular}

FIGURE 7 Example of modified Haar transformation matrix for $n=3$.

fact, this decrease in the dimension of the range space corresponds directly to various Shannon co-factors of the function to be transformed.

The output vector of the function to be transformed generally contains integers with -1 representing logic-1 and +1 representing logic- 0 . With this viewpoint, we can define the number of matches between a particular transformation matrix row vector as the number of times the row vector and function vector components are simultaneously equal to -1 or +1 . Since some of the rows represent functions that are masked by co-factors, the row-function space is less than $2^{3}$ in size and the presence of a 0 value acts as a place holder.

The presence of co-factors in the Haar constituent functions can be accounted for by using Bayes' theorem to represent these quantities as output probabilities of the AND of the function to be transformed with its respective dependent literals. These functions are shown to the left of the transformation matrix in Fig. 7.

In order to determine the total number of matching outputs between $f$ and a row-function, it is necessary to determine when both simultaneously evaluate to a logic-0 level as well as a logic-1 level. We denote the percentage of the total number of matches of logic- 0 between some $f$ and a row-function as $p_{m 0}$ and likewise for the logic-1 levels, $p_{m 1}$. With this viewpoint, the composite $f_{c}$ expressions can be constructed (shown to the left of the transformation matrix in Fig. 7 that utilize co-factors of the function to be transformed to restrict the range space and to dictate where the relative location of the valid output of the $f_{c}$ function occurs in the $2^{n}$ row vector components.

Given these observations, we see that the $k$-th modified Haar spectral coefficient can be calculated as:

$$
H_{k}=2^{n-i}\left[2\left(p_{m 0}+p_{m 1}\right)-1\right]
$$

where $n$ is the dimension of the range space of the function to be transformed, $f$, and $i$ is the dimension of the range space of a particular Shannon co-factor of $f$. If $N_{m}$ represents the number of times an intermediate product value of +1 occurs in the computation of a particular modified Haar spectral coefficient (corresponding to $1 \times 1$ and $-1 \times-1$ products) and $N_{m m}$ corresponds to the number of times a product value of -1 occurs (corresponding to $1 \times-1$ products), then the $k$-th 
TABLE I Relationship of the Haar spectrum and output probabilities

\begin{tabular}{lccll}
\hline Symbol & $i$ & $n-i$ & \multicolumn{1}{c}{$p_{m 1}$} & \multicolumn{1}{c}{$p_{m 0}$} \\
\hline$H_{0}$ & 0 & 3 & $P[f \cdot 0]$ & $P[\bar{f} \cdot \overline{0}]$ \\
$H_{1}$ & 0 & 3 & $P\left[f \cdot x_{1}\right]$ & $P\left[\bar{f} \cdot \bar{x}_{1}\right]$ \\
$H_{2}$ & 1 & 2 & $P\left[f \cdot \bar{x}_{1} \cdot x_{2}\right] / P\left[\bar{x}_{1}\right]$ & $P\left[\bar{f} \cdot \bar{x}_{1} \cdot \bar{x}_{2}\right] / P\left[\bar{x}_{1}\right]$ \\
$H_{3}$ & 1 & 2 & $P\left[f \cdot x_{1} \cdot x_{2}\right] / P\left[x_{1}\right]$ & $P\left[\bar{f} \cdot x_{1} \cdot \bar{x}_{2}\right] / P\left[x_{1}\right]$ \\
$H_{4}$ & 2 & 1 & $P\left[f \cdot \bar{x}_{1} \cdot \bar{x}_{2} \cdot x_{3}\right] / P\left[\bar{x}_{1} \cdot \bar{x}_{2}\right]$ & $P\left[\bar{f} \cdot \bar{x}_{1} \cdot \bar{x}_{2} \cdot \bar{x}_{3}\right] / P\left[\bar{x}_{1} \cdot \bar{x}_{2}\right]$ \\
$H_{5}$ & 2 & 1 & $P\left[f \cdot \bar{x}_{1} \cdot x_{2} \cdot x_{3}\right] / P\left[\bar{x}_{1} \cdot x_{2}\right]$ & $P\left[\bar{f} \cdot \bar{x}_{1} \cdot x_{2} \cdot \bar{x}_{3}\right] / P\left[\bar{x}_{1} \cdot x_{2}\right]$ \\
$H_{6}$ & 2 & 1 & $P\left[f \cdot x_{1} \cdot \bar{x}_{2} \cdot x_{3}\right] / P\left[x_{1} \cdot \bar{x}_{2}\right]$ & $P\left[\bar{f} \cdot x_{1} \cdot \bar{x}_{2} \cdot \bar{x}_{3}\right] / P\left[x_{1} \cdot \bar{x}_{2}\right]$ \\
$H_{7}$ & 2 & 1 & $P\left[f \cdot x_{1} \cdot x_{2} \cdot x_{3}\right] / P\left[x_{1} \cdot x_{2}\right]$ & $P\left[\bar{f} \cdot x_{1} \cdot x_{2} \cdot \bar{x}_{3}\right] / P\left[x_{1} \cdot x_{2}\right]$ \\
\hline
\end{tabular}

modified Haar spectral coefficient is given as:

$$
H_{k}=N_{m}-N_{m m}
$$

It is noted that the sum of $N_{m}$ and $N_{m m}$ must necessarily equal $2^{n-i}$ where $i$ indicates the number of variables about which co-factors have been taken. Substituting this observation into Eq. (5) yields:

$$
H_{k}=2 N_{m}-2^{n-i}
$$

We define $p_{m}$ to be the total percentage of times that a matching output between the $f$ and $f_{c}$ functions occur, therefore $p_{m}=2^{n-i} \times N_{m}$. Furthermore, $p_{m}=p_{m 0}+p_{m 1}$. Substituting these definitions into Eq. (6) yields the result:

$$
H_{k}=2^{n-i}[2 p m-1]
$$

The result of Eq. (7) reduces the computation of a single modified Haar spectral coefficient to that of finding matching percentages of identical similar outputs of $f$ and a transformation matrix row-function. This can be accomplished by applying the output probability computation algorithm to an OBDD representation of the $f_{c}$ functions. Using the result of Bayes' theorem, the cofactor output probabilities can be computed by ANDing various cubes with the original function $f$ and dividing the result by the output probability of the cube itself, which is a constant.

Table I contains symbols for each of the Haar spectral coefficients $\left(H_{i}\right)$ values that indicate the size of the cofactor function range $(i)$ and probability expressions that evaluate whether the function to be transformed and the row function simultaneously evaluate to logic-0 (denoted as $p_{m 0}$ ), or evaluate to logic-1 (denoted as $\left.p_{m 1}\right)$.

By observing that the $p_{m 0}$ and $p_{m 1}$ expressions for a given $H_{k}$ in Table I are statistically independent, the individual computations may be combined into a compact form. As an example, consider $H_{5}$.

Example 2 The divisor for the $p_{m 0}$ and $p_{m 1}$ expressions, $P\left[\bar{x}_{1} x_{2}\right]$ is a constant equal to $1 / 2^{i}$ and thus may be factored out resulting in Eq. (4) being rewritten as:

$$
H_{k}=2^{n-i}\left[2^{i+1}\left(p_{m 0}+p_{m 1}\right)-1\right]
$$

Since the Boolean expressions $f \cdot \bar{x}_{1} \cdot x_{2} \cdot x_{3}$ and $\bar{f} \cdot \bar{x}_{1} \cdot x_{2} \cdot \bar{x}_{3}$ are disjoint, the overall probability may be computed as the sum of the individual probabilities, or alternatively, as the probability of the inclusive-OR of the functions. This is true because it is easy to see that $P[g+h]=$ $P[g]+P[h]$ for $g$ and $h$ that are covered by disjoint cube sets.

Combining the Boolean arguments and simplifying:

$$
f \cdot \bar{x}_{1} \cdot x_{2} \cdot x_{3}+\bar{f} \cdot \bar{x}_{1} \cdot x_{2} \cdot \bar{x}_{3}=\bar{x}_{1} x_{2}\left(\overline{x_{3} \oplus f}\right)
$$

Therefore, we can rewrite Eq. (8) as:

$$
H_{k}=2^{n-i}\left[2^{i+1} P\left[\bar{x}_{1} x_{2}\left(\overline{x_{3} \oplus f}\right)\right]-1\right]
$$

The manipulations used in Example 2 may be applied to all of the modified Haar spectrum coefficients. This leads to the interesting result that the modified Haar coefficients depend on the set of $n+1$ Boolean relations, $\left\{\overline{f \oplus 0}, \overline{f \oplus x_{1}}, \overline{f \oplus x_{2}}, \cdots \overline{f \oplus x_{n}}\right\}$, which describe the equivalence of a particular dependent variable, $x_{i}$, and the function to be transformed, $f$. We refer to this set of functions as the characteristic equivalence relations. Higher ordered coefficients are based on disjoint partitions of the range space of these equivalence functions. The partitioning is accomplished by ANDing the equivalence functions with various cubes of other dependent variables of $f$ referred to as the characteristic cubes. The specific cofactor that $p_{m}$ is computed from is given by the inherent order of the dependent variables describing $f$.

Table II contains the probability functions for an $n=3$ variable transformation in terms of the characteristic equivalence relations. Using this table, each coefficient can be computed using Eqs. (11) and (12).

$$
\begin{gathered}
H_{i}=2^{n-j}\left[2^{j+1} p_{m}-1\right] \\
j=\left\{\begin{array}{ll}
0, & i=0 \\
\left\lfloor\log _{2}(i)\right\rfloor, & i>0
\end{array}\right)
\end{gathered}
$$

We can also compute the total number of possible different valued coefficients for a particular $i$ (or equivalently, a particular $j$ ). We note that the Haar coefficients range in value as given by $\left\{-2^{n-j},-2^{n-j}+2,-2^{n-j}+\right.$ $\left.4, \ldots,-2,0,+2, \ldots, 2^{n-j}-4,2^{n-j}-2,2^{n-j}\right\}$. Thus, the total number of possible different valued coefficients (denoted by $N_{j}$ ) is given in Eq. (13).

$$
N_{j}=2^{n-j}+1
$$

\section{Probabilistic Equivalence Checking}

By the definition of event $E$ and the assumption that all functions of $n$ variables are equally likely to arise (uniform distribution), it is easy to see that:

$$
P[E]=\frac{1}{2^{2^{n}}}
$$

Since the Modified Haar spectrum for a given fully specified Boolean function is unique [11], Eq. (15) also 
TABLE II Relationship of the Haar spectrum and characteristic equivalence functions

\begin{tabular}{llcl}
\hline Symbol & $j$ & $n-j$ & \multicolumn{1}{c}{$p_{m}$} \\
\hline$H_{0}$ & 0 & 3 & $P[\overline{0} \cdot \overline{f \oplus 0}]$ \\
$H_{1}$ & 0 & 3 & $P\left[\overline{0} \cdot \overline{f \oplus x_{1}}\right]$ \\
$H_{2}$ & 1 & 2 & $P\left[\bar{x}_{1} \cdot \overline{f \oplus x_{2}}\right]$ \\
$H_{3}$ & 1 & 2 & $P\left[x_{1} \cdot \bar{f} \oplus x_{2}\right]$ \\
$H_{4}$ & 2 & 1 & $P\left[\bar{x}_{1} \cdot \bar{x}_{2} \cdot \bar{f} \oplus x_{3}\right]$ \\
$H_{5}$ & 2 & 1 & $P\left[\bar{x}_{1} \cdot x_{2} \cdot \overline{f \oplus x_{3}}\right]$ \\
$H_{6}$ & 2 & 1 & $P\left[x_{1} \cdot \bar{x}_{2} \cdot \bar{f} \oplus x_{3}\right]$ \\
$H_{7}$ & 2 & 1 & $P\left[x_{1} \cdot x_{2} \cdot \bar{f} \oplus x_{3}\right]$ \\
\hline
\end{tabular}

holds.

$$
P\left[S_{i} \mid E\right]=1
$$

Equation (15) may be generalized for the occurrence of any subset of $q$ events, $\left\{S_{i}\right\}$, to that shown in Eq. (16).

$$
P\left[\bigcap_{i=1}^{q} S i \mid E\right]=1
$$

Also we see that $P\left[S_{i}\right]$ is the ratio of all possible functions that yield the coefficient, $H_{i}(f)$, divided by the total population of $2^{2^{n}}$. We define a counting function, $k\left(H_{i}\right)$, that is integer valued and yields the number of fully specified Boolean functions for which the $i$-th Haar spectral coefficient is $H_{i}$. Thus we can express this relationship as shown in Eq. (17).

$$
P\left[S_{i}\right]=\frac{k\left(H_{i}\right)}{2^{2^{n}}}
$$

From probability theory, we know that Eq. (18) holds.

$$
P\left[E \bigcap S_{i}\right]=P\left[S_{i} \mid E\right] P[E]=P\left[E \mid S_{i}\right] P\left[S_{i}\right]
$$

Using the relationships in Eqs. (14), (16) and (18), we see that the conditional probability becomes:

$$
P\left[E \mid S_{i}\right]=\frac{P[E]}{P\left[S_{i}\right]}=\frac{1}{k\left(H_{i}\right)}
$$

In general, for any subset of events, $\left\{S_{i}\right\}$, we have the expression as given in Eq. (20).

$$
\begin{aligned}
P\left[E \mid \bigcap_{i=1}^{q} S_{i}\right] & =\frac{P\left[E \bigcap\left(\bigcap_{i=1}^{q} S_{i}\right)\right]}{P\left[\bigcap_{i=1}^{q} S_{i}\right]} \\
& =\left(\frac{1}{2^{2^{n}}}\right)\left(\frac{1}{P\left[\bigcap_{i=1}^{q} S_{i}\right]}\right) \\
& =\frac{1}{2^{2^{n}} P\left[\bigcap_{i=1}^{q} S_{i}\right]}
\end{aligned}
$$

Equation (20) is the governing expression for the probabilistic equivalence checking technique described in this paper. We see that given a subset of matching Haar spectral coefficients for two functions, $f$ and $g$, (or alternatively, a subset of events, $\left\{S_{i}\right\}$ ), the probability that $f$ and $g$ are indeed equivalent may be computed. By obtaining the information that a new event $S_{i}$ has occurred, we may update the value $P\left[\bigcap_{i=1}^{q} S_{i}\right]$ thereby increasing the value $P\left[E \mid \bigcap_{i=1}^{q} S_{i}\right]$.

\section{Relation of Haar Coefficients to Probabilistic Events}

This section will derive the relationship between the probabilistic events, $S_{i}$, and their dependence upon the corresponding Haar spectral coefficients, $H_{i}(f)$ and $H_{i}(g)$. The Haar spectral coefficients may be obtained through the use of any efficient method such as those in Refs. $[8,9,18]$.

We note that given the $i$-th Haar spectral coefficient for a function, $f$, and a function, $g$, there appear to be four possibilities as given in Table III. It is seen that as soon as $H_{i}(f) \neq H_{i}(g)$ occurs, it is possible to declare $f \neq g$ and to terminate the process of equivalence checking. However, when $H_{i}(f)=H_{i}(g)$, it is not known whether $f=g$ or $f \neq g$ unless all possible $H_{i}$ are found to be equivalent. However, it is possible to successively refine the $P\left[E \mid \bigcap_{i=1}^{q} S_{i}\right]$ value using Eq. (20).

For this probabilistic scheme to be practically useful, we need to determine the joint distribution, $P\left[\bigcap_{i}^{j<2^{n}-1} S_{i}\right.$, as a function of the corresponding subset of Haar spectral coefficients. We first consider the simple case of determining a function for $P\left[S_{i}\right]$ that depends on the single Haar spectral coefficient, $H_{i}$. For a single matching coefficient, we are interested in finding, $P\left[E \mid S_{i}\right]$. Since it is known that $P\left[E \cap S_{i}\right]=P\left[S_{i}\right] P\left[E \mid S_{i}\right]$, we can express the conditional probability as given in Eq. (21) since $P\left[S_{i}\right] \neq 0$.

$$
P\left[E \mid S_{i}\right]=\frac{P\left[E \cap S_{i}\right]}{P\left[S_{i}\right]}
$$

The numerator of Eq. (21) is the percentage of functions $f$ and $g$ that have a common Haar coefficient, $H_{i}$. Since all equivalent functions have the same Haar spectra by the uniqueness property of the transform, we see that $P[E \cap$ $\left.S_{i}\right]=1 / 2^{2^{n}}$. The denominator of Eq. (21) is the percentage of functions that have a common $H_{i}$ value. In general, many different functions can have common $H_{i}$ values. For example, 6 out 16 possible functions of $n=2$ variables have $H_{0}=0$. Based on the definition of the counting function, $k\left(H_{i}\right)$, we can then express $P\left[S_{i}\right]=k\left(H_{i}\right) / 2^{2^{n}}$ and

TABLE III Apparent possibilities given $f, g, H_{i}(f)$ and $H_{i}(g)$

\begin{tabular}{lll}
\hline Function relation & $H_{i}$ relation & Observation \\
\hline$f=g$ & $H_{i}(f)=H_{i}(g)$ & Possible $f=g$ \\
$f=g$ & $H_{i}(f) \neq H_{i}(g)$ & Not possible \\
$f \neq g$ & $H_{i}(f)=H_{i}(g)$ & Possible $f=g$ \\
$f \neq g$ & $H_{i}(f) \neq H_{i}(g)$ & $f \neq g$ \\
\hline
\end{tabular}


Eq. (21) is rewritten as Eq. (22).

$$
P\left[E \mid S_{i}\right]=\frac{1}{k\left(H_{i}\right)}
$$

The relationship between the characteristic equivalence functions and the Haar spectral coefficients is established in the following results.

Lemma 1 Two Boolean functions, $f\left(x_{1}, x_{2}, \ldots, x_{n}\right)$ and $g\left(x_{1}, x_{2}, \ldots, x_{n}\right) \quad$ cannot be equivalent if it is true that $P\left[\overline{f \oplus x_{i}}\right] \neq P\left[\overline{g \oplus x_{i}}\right]$.

Proof Assume the contradiction of the lemma, that is $P\left[\overline{f \oplus x_{i}}\right] \neq P\left[\overline{g \oplus x_{i}}\right]$, but $f=g$. Since $f=g$, then it must be true that $\overline{f \oplus x_{i}}=\overline{g \oplus x_{i}}$ and that $P\left[\overline{f \oplus x_{i}}\right]=m_{f} / 2^{n}$ and $P\left[\overline{g \oplus x_{i}}\right]=m_{g} / 2^{n}$ where $m_{f}$ is the number of distinct 1values in the truth vector of $\overline{f \oplus x_{i}}$ and $m_{g}$ is the number of distinct 1 -values in the truth vector of $\overline{g \oplus x_{i}}$. But since $P[f]=P[g]$ and $\overline{f \oplus x_{i}}=\overline{g \oplus x_{i}}$, it must be the case that $m_{f}=m_{g}$, thus contradicting the assumption that $P\left[\overline{f \oplus x_{i}}\right] \neq P\left[\overline{g \oplus x_{i}}\right]$.

COROLlary 1 Two co-factors about the same cube of $\overline{f \oplus x_{i}}$ and $\overline{g \oplus x_{i}}$ have identical output probabilities.

We denote $f_{c i}$ as the function that is formed as the intersection of some cube and $i$-th characteristic equivalence function. Thus, $f_{c i}$ depends upon all $n$ variables and $p_{m}=P\left[f_{c i}\right]$. The total number of functions with a common $H_{i}$ value (denoted as $k\left(H_{i}\right)$ ), can be computed as the total number of different $f_{c i}$ functions that have a common $P\left[f_{c i}\right]$ due to Corollary 1 . Therefore, $k\left(H_{i}\right)$ is the different number of ways that a function with a range space of size $2^{n-j}$ can have $2^{n} p_{m}$ logic- 1 values. This combinatorial quantity must be scaled by a constant to account for the decreasing magnitude of the $H_{i}$ that are distributed over the entire population of $2^{2^{n}}$ functions as $i$ increases. This scaling factor is seen to be $2^{N_{0}}-N_{j}$ where $N_{i}$ is defined in Eq. (13). Given these observations, $k\left(H_{i}\right)$ can be expressed as:

$$
k\left(H_{i}\right)=2^{N_{0}-N_{j}}\left(\begin{array}{c}
2^{n-j} \\
2^{n} p_{m}
\end{array}\right)
$$

Using the fact that $2^{N_{0}-N_{j}}=2^{2^{n}-2^{n-j}}$ and that $p_{m}=$ $\left(H_{i}+2^{n-j}\right) /\left(2^{n+1}\right)$, we can reduce Eq. (23) to Eq. (24).

$$
k\left(H_{i}\right)=2^{2^{n}-2^{n-j}}\left(\begin{array}{c}
2^{n-j} \\
\frac{H_{i}+2^{n-j}}{2}
\end{array}\right)
$$

Thus, we can rewrite Eq. (22) as shown in Eq. (25).

$$
P\left[E \mid S_{i}\right]=\left(\frac{1}{2^{2^{n}-2^{n-j}}}\right)\left(\begin{array}{c}
2^{n-j} \\
\frac{H_{i}+2^{n-j}}{2}
\end{array}\right)^{-1}
$$

As an example, consider the case where $H_{0}(f)=$ $H_{0}(g)=2$ for $n=2$ variables. To compute $P\left[E \mid S_{0}\right]$, we use the relationship in Eq. (25) resulting in $P\left[E \mid S_{0}\right]=1 / 4$.

To successively improve the $P\left[E \mid S_{i}\right]$ value, it must be updated with each subsequent event, $S_{i}$. For a practical implementation, this means that $P\left[\bigcap_{i}^{j<2^{n}-1} S_{i}\right]$ must be computed as a function of the corresponding Haar coefficients, $H_{i}$. However, this value cannot be computed as a simple product of individual $P\left[S_{i}\right]$ values since the multiple $S_{i}$ events are not necessarily statistically independent.

For the general case, we must re-compute $P\left[\bigcap_{i=1}^{q} S_{i}\right]$ for each new event, $S_{i}$, in order to update the $P\left[E \mid \bigcap_{i=1}^{q} S_{i}\right]$ value. Some events are statistically dependent while other subsets are not. Recall that the values, $H_{i}$, depend on cofactor functions of various characteristic equivalence functions about some cube. A subset of events, $\left\{S_{i}\right\}$, are all statistically independent if they result from a corresponding subset of matching Haar spectral coefficients, $\left\{H_{i}\right\}$, that are formed based on Shannon co-factors with respect to mutually disjoint cubes. As an example, $\mathrm{H}_{2}$ and $\mathrm{H}_{3}$, result from the functions $\bar{x}_{1} \cdot \overline{f \oplus x_{2}}$ and $x_{1} \cdot \overline{f \oplus x_{2}}$ which are disjoint. Thus, $P\left[S_{2} \bigcap S_{3}\right]=P\left[S_{2}\right] P\left[S_{3}\right]$ since $\bar{x}_{1}$ and $x_{1}$ are disjoint characteristic cubes.

Not all events, $\left\{S_{i}\right\}$, are statistically independent. As an example, $H_{0}$ and $H_{1}$ are dependent since an intersection of the co-factors of the characteristic equivalence functions of $H_{0}$ and $H_{1}$ exists and is non-null. In order to find the value $P\left[S_{1} \cap S_{0}\right]$, we generalize our definition of the counting function to $k\left(H_{0}, H_{1}\right)$ which will denote the number of possible Boolean functions that may have both $H_{0}$ and $H_{1}$ as Haar spectral coefficients. Given this quantity, we may then express the desired joint probability as given in Eq. (26).

$$
P\left[S_{0} \bigcap S_{1}\right]=\frac{k\left(H_{0}, H_{1}\right)}{2^{2^{n}}}
$$

In general, we have Eq. (27) resulting in Eq. (28).

$$
\begin{gathered}
P\left[\bigcap_{i=0}^{q} S_{i}\right]=\frac{k\left(H_{i}, H_{i+1}, \ldots, H_{q}\right)}{2^{2^{n}}} \\
P\left[E \mid \bigcap_{i=0}^{q} S_{i}\right]=\frac{1}{2^{2^{n}} P\left[\bigcap_{i=1}^{q} S_{i}\right]}=\frac{1}{k\left(H_{i}, H_{i+1}, \ldots, H_{q}\right)}
\end{gathered}
$$

To compute this joint probability distribution, we must have some information concerning the dependent relationship between individual $k\left(H_{i}\right)$ and $k\left(H_{m}\right)$ values. As an example of this dependence, we will derive the relationship between $H_{0}$ and $H_{1}$. For $i=0,1$, the integer $j$ is zero valued yielding the relationships as shown in Eqs. (29) and (30).

$$
\begin{aligned}
& H_{0}=2^{n}\left[2 p_{m 0}-1\right] \\
& H_{1}=2^{n}\left[2 p_{m 1}-1\right]
\end{aligned}
$$

For these two coefficients, we have the output probabilities $p_{m 0}$ and $p_{m 1}$ that may be expressed as:

$$
\begin{gathered}
p_{m 0}=P[\overline{0} \cdot \bar{f} \oplus 0]=P[\bar{f}]=\frac{1}{2}\left\{P\left[\bar{f}_{\bar{x}_{1}}\right]+P\left[\bar{f}_{x_{1}}\right]\right\} \\
p_{m 1}=P\left[\overline{0} \cdot \overline{f \oplus x_{1}}\right]=P\left[\overline{f \oplus x_{1}}\right]=\frac{1}{2}\left\{P\left[f_{x_{1}}\right]+P\left[\bar{f}_{\bar{x}_{1}}\right]\right\}
\end{gathered}
$$


Thus, the corresponding Haar spectral coefficients become:

$$
\begin{aligned}
& H_{0}=2^{n}\left\{P\left[\bar{f}_{\bar{x}_{1}}\right]+P\left[\bar{f}_{x_{1}}\right]-1\right\} \\
& H_{1}=2^{n}\left\{P\left[\bar{f}_{\bar{x}_{1}}\right]+P\left[f_{x_{1}}\right]-1\right\}
\end{aligned}
$$

Equating the $P\left[\bar{f}_{\bar{x}_{1}}\right]$ values results in the relationship between $H_{0}$ and $H_{1}$ as given in Eq. (35).

$$
H_{1}=H_{0}+2^{n}\left\{P\left[f_{x_{1}}\right]-P\left[\bar{f}_{x_{1}}\right]\right\}
$$

Using the result of Eqs. (24) and (35), we have:

$$
\begin{aligned}
k\left(H_{1}\right) & =\left(\begin{array}{c}
2^{n} \\
\frac{H_{1}+2^{n}}{2}
\end{array}\right)=\left(\begin{array}{c}
2^{n} \\
\frac{H_{0}+2^{n}\left\{P\left[f_{x_{1}}\right]-P\left[\bar{f}_{x_{1}}\right]\right\}+2^{n}}{2}
\end{array}\right) \\
& =\left(\begin{array}{c}
2^{n} \\
\frac{H_{0}+2^{n}}{2}+\frac{H_{1}-H_{0}}{2}
\end{array}\right)
\end{aligned}
$$

Using the identity relation:

$$
\left(\begin{array}{l}
n \\
k
\end{array}\right)\left(\begin{array}{c}
n-k \\
m-k
\end{array}\right)=\left(\begin{array}{l}
n \\
m
\end{array}\right)\left(\begin{array}{l}
m \\
k
\end{array}\right)
$$

We rewrite Eq. (36) as:

$$
\left(\begin{array}{c}
2^{n} \\
\frac{H_{0}+2^{n}}{2}
\end{array}\right)\left(\begin{array}{c}
\frac{H_{0}+2^{n}}{2} \\
\frac{H_{1}+2^{n}}{2}
\end{array}\right)=\left(\begin{array}{c}
2^{n}-\frac{H_{1}+2^{n}}{2} \\
\frac{H_{0}+2^{n}}{2}-\frac{H_{1}+2^{n}}{2}
\end{array}\right)\left(\begin{array}{c}
2^{n} \\
\frac{H_{1}+2^{n}}{2}
\end{array}\right)
$$

Equation (38) reduces to Eq. (39) and we define the resulting quantity as the value $A_{0,1}$.

$$
A_{0,1}=\frac{k\left(H_{0}\right)}{k\left(H_{1}\right)}=\left(\begin{array}{c}
2^{n}-\frac{H_{1}+2^{n}}{2} \\
\frac{H_{0}+2^{n}}{2}-\frac{H_{1}+2^{n}}{2}
\end{array}\right)\left(\begin{array}{c}
\frac{H_{0}+2^{n}}{2} \\
\frac{H_{1}+2^{n}}{2}
\end{array}\right)^{-1}
$$

Thus, we have the result that the values $k\left(H_{0}\right)$ and $k\left(H_{1}\right)$ are deterministically related as $k\left(H_{0}\right)=A_{0,1} k\left(H_{1}\right)$. Using this fact, we devise a means for computing the desired joint quantity, $k\left(H_{0}, H_{1}\right)$. We know that for a given $H_{0}$ and $H_{1}$ to exist for a single function, the corresponding $k\left(H_{0}\right)-A_{0,1} k\left(H_{1}\right)=0$. Thus, it is possible to check all possible $k\left(H_{1}\right)$ values for $H_{1}=\left\{-2^{n},-2^{n}+\right.$ $\left.2, \ldots,-2,0,2, \ldots, 2^{n}-2,2^{n}\right\}$ and where the relationship is satisfied, we increment the value of $k\left(H_{0}, H_{1}\right)$. This may be expressed in closed form through the use of the unitstep function, $u(\tau)$, as defined in Eq. (40).

$$
u(\tau)= \begin{cases}1, & \tau=0 \\ 0, & \text { otherwise }\end{cases}
$$

Equation (41) then expresses the desired relationship.

$$
k\left(H_{0}, H_{1}\right)=\sum_{i=0}^{2^{n}+1} u\left[k\left(H_{0}\right)-A_{0,1} k\left(H_{i}\right)\right]
$$

The upper bound of the summation is the total number of possible Haar spectral coefficients that can result for the $i=1$ coefficient. Although the complexity of this approach is prohibitive for low-ordered coefficients, Eq. (41) can be algorithmically stated as follows:

1. Compute $A_{0,1}$ based on the value of the Haar spectral coefficients.

2. Initialize $k\left(H_{0}, H_{1}\right)=0$.

3. Iterate over all possible $2^{n}+1$ values of $H_{1}$ and evaluate if $\left(k\left(H_{0}\right)-A_{0,1} k\left(H_{1}\right)==0\right)$ then $k\left(H_{0}, H_{1}\right)++$

4. $P\left[S_{0} \cap S_{1}\right]=\left(k\left(H_{0}, H_{1}\right)\right) / 2^{2^{n}}$, or, $P\left[E \mid\left(S_{0} \cap S_{1}\right)\right]=$ $1 /\left(k\left(H_{0}, H_{1}\right)\right)$.

This procedure can be generalized by deriving and computing new $A_{i, j, \ldots, k}$ values each time a new event, $S_{k}$, occurs and updating the $P\left[\bigcap_{i=1}^{q} S_{i}\right]$ and hence, the $P\left[E \mid \bigcap_{i=1}^{q} S_{i}\right]$ values.

In general, this procedure leads to exponential complexity for updating all new $k$ values. However, we note that the multi-resolution nature of the Haar transform allows us to determine subsets of coefficients that are statistically independent, thus avoiding the computation of large joint distributions. This can also be coupled with the construction of the partial BDDs by constraining them to represent mutually disjoint portions of the functions under consideration. We also note that the decreased range space dimension of high-ordered coefficients, $H_{i}$, can allow the algorithm to run in reasonable time for those $H_{i}$.

\begin{tabular}{|c|c|c|c|c|c|}
\hline Function $(f)$ & $H_{0}$ & $H_{1}$ & $\mathrm{H}_{2}$ & $\mathrm{H}_{3}$ & Expression \\
\hline 0000 & 4 & 0 & 0 & 0 & 0 \\
\hline 0001 & 2 & 2 & 0 & 2 & $x y$ \\
\hline 0010 & 2 & 2 & 0 & -2 & $x \bar{y}$ \\
\hline 0011 & 0 & 4 & 0 & 0 & $x$ \\
\hline 0100 & 2 & -2 & 2 & 0 & $\bar{x} y$ \\
\hline 0101 & 0 & 0 & 2 & 2 & $y$ \\
\hline 0110 & 0 & 0 & 2 & -2 & $x \oplus y$ \\
\hline 0111 & -2 & 2 & 2 & 0 & $x \pm y$ \\
\hline 1000 & 2 & -2 & -2 & 0 & $\overline{x y}$ \\
\hline 1001 & 0 & 0 & -2 & 2 & $\overline{x \oplus y}$ \\
\hline 1010 & 0 & 0 & -2 & -2 & $\bar{y}$ \\
\hline 1011 & -2 & 2 & -2 & 0 & $x+\bar{y}$ \\
\hline 1100 & 0 & -4 & 0 & 0 & $\bar{x}$ \\
\hline 1101 & -2 & -2 & 0 & 2 & $\bar{x}+y$ \\
\hline 1110 & -2 & -2 & 0 & -2 & $\bar{x}+\bar{y}$ \\
\hline 1111 & -4 & 0 & 0 & 0 & 1 \\
\hline
\end{tabular}

\section{EXAMPLE CALCULATION}

As an example, consider Table IV which contains the Haar spectral vectors for all possible functions of $n=2$ variables. We will assume that we are dealing with two functions, $f\left(x_{1}, x_{2}\right)$ and $g\left(x_{1}, x_{2}\right)$ such that $f$ and $g$ are equivalent to the function represented in the third row of Table IV. Thus, the corresponding Haar spectral vector

TABLE IV All possible Boolean functions for $n=2$ and their Haar spectra 

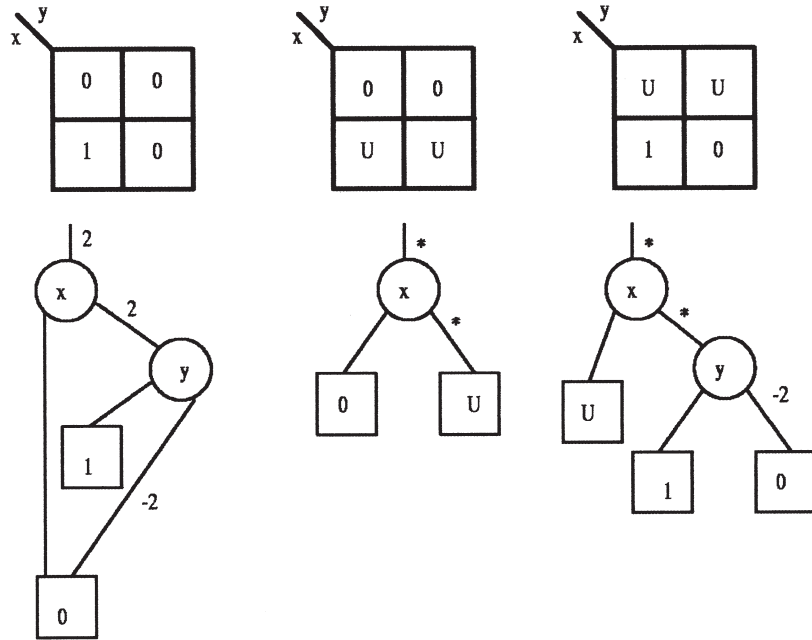

FIGURE 8 Karnaugh maps and HSD/BDDs of complete and partial functions.

is $\quad \underline{H}^{T}(f)=\underline{H}^{T}(g)=\left(H_{0}, H_{1}, H_{2}, H_{3}\right)=(2,2,0,-2)$. Figure 8 contains the Karnaugh maps and corresponding partial and complete BDDs for the function $f$ (or $g$ ). Note that the BDDs are also interpreted as HSDs with the 1edges having an attribute equal to a Haar spectral coefficient value. The coefficient attributes are shown on the HSD/BDDs with an "*" indicating that the exact coefficient could not be computed. From the center partial HSD/BDD, we see that $H_{2}=0$ and from the rightmost partial HSD/BDD we see that $H_{3}=-2$.

From the partial BDDs, it is seen that only two Haar spectral coefficients can be obtained, $H_{2}$ and $H_{3}$. This is due to the fact that $H_{0}$ and $H_{1}$ require a completely specified HSD since the corresponding transform matrix rows have no 0 -valued entries. For more practical cases with much larger values of $n$, we obtain a larger fraction of the total number of Haar coefficients than the 50\% obtained from this small example.

Using the previously derived equations, we have $k\left(H_{2}\right)=8, k\left(H_{3}\right)=4$ and $k\left(H_{2}, H_{3}\right)=2$. These values result in the probability values $P\left[E \mid S_{2}\right]=1 / 8, P\left[E \mid S_{3}\right]=$ $1 / 4$ and $P\left[E \mid\left(S_{2} \cap S_{3}\right)\right]=1 / 2$. Furthermore, we note that $P\left[S_{2} \cap S_{3}\right]=P\left[S_{2}\right] P\left[S_{3}\right]=(1 / 2)(1 / 4)=1 / 8$ in this case since $S_{2}$ and $S_{3}$ are statistically independent. The independence arose from the fact that the two partial BDDs represent disjoint segments of the range space of the function. If this is ensured during the construction of all partial BDDs, the joint computation of $k\left(H_{2}, H_{3}\right)$ may be avoided and $P\left[E \mid\left(S_{2} \cap S_{3}\right)\right]$ may be computed as given in Eq. (42).

$$
\begin{aligned}
P\left[E \mid\left(S_{2} \cap S_{3}\right)\right] & =\frac{1}{2^{2^{n}} P\left[S_{2} \cap S_{3}\right]}=\frac{1}{2^{2^{n}} P\left[S_{2}\right] P\left[S_{3}\right]} \\
& =\frac{1}{16(1 / 8)}=\frac{1}{2}
\end{aligned}
$$

This result shows that there are only two possible functions out of the population of $2^{2^{n}}=16$ that have $H_{2}=$ 0 and $H_{3}=-2$.

\section{EXPERIMENTAL RESULTS}

Experiments were formulated to investigate the effectiveness of using Haar spectral coefficients for equivalence checking. These experiments were run to observe the average number of Haar coefficients needed before a mismatch in value was found for two functions known to be slightly different. The results also give an indication of how different errors between two versions of a circuit affect the number of required Haar coefficients for a mismatch to be found.

The initial set-up for this experiment involved choosing a single output from a benchmark function and randomly inserting a single inverter in the netlist. Next, HSDs were formed for the circuit with the inverter and without. To ensure the two HSDs did indeed represent different functions, a graphical equivalence checker was used. The experiment consisted of randomly extracting pairs of same-order Haar coefficients from the two representations until two were found that differed in value. For each given circuit error (that is, each given inverter insertion) 1024 trials were made.

Table V contains the results for 10 benchmark functions, each with 10 different inverter errors. The column labeled Inp contains the number of distinct variables that the function depends on and the row labeled $a v g$ is the average number of Haar coefficients (over the 1024 trials) that were required before a mismatch occurred. Likewise, the row labeled dev contains the standard deviation of the number of required Haar coefficients. It is apparent that the standard deviation is approximately the same value as the mean in all cases. This is a result of the fact that the subset of Haar coefficients was chosen randomly with the assumption that each was equally likely for two designs that are known to differ (i.e. a geometric distribution resulted in terms of the average number of coefficients before a mismatch occurred). Although this observation is largely an artifact of our experimental setup, another result is the large range in value of the required number of coefficients in order to detect the differences in the two circuits. As an example, we see that the benchmark frgl has differences in the averages that are as great as four orders of magnitude (e.g. 70.1 versus 104225.8).

The data presented in Table VI was computed in order to compare the Haar coefficient matching scheme to random simulations. These results compare the average number of required Haar coefficients to the number of random simulations that must be performed before a difference in the two circuits is detected. The simulations were performed using equally likely, randomly generated test vectors. The averages were formed over the 10 circuit modifications described above with 1024 trials each. In 
TABLE V Effect of different errors on Haar coefficient matching

\begin{tabular}{|c|c|c|c|c|c|c|c|c|c|c|c|c|}
\hline \multirow{3}{*}{$\frac{\text { Circuit }}{\text { c432 }}$} & \multirow{3}{*}{$\begin{array}{r}\text { Inp } \\
36\end{array}$} & \multicolumn{11}{|c|}{ Inverter error (10 random trials) } \\
\hline & & avg & 58.0 & 24.8 & 474.1 & 15.9 & 58.3 & 9.6 & 8.2 & 8.4 & 97.5 & 56.4 \\
\hline & & dev & 61.3 & 24.5 & 471.2 & 15.4 & 55.3 & 9.2 & 8.2 & 8.4 & 97.5 & 56.4 \\
\hline \multirow[t]{2}{*}{ c499 } & 41 & avg & 69.3 & 65.3 & 61.4 & 68.3 & 66.1 & 165.6 & 59.2 & 59.5 & 61.4 & 66.4 \\
\hline & & dev & 70.4 & 66.6 & 60.1 & 66.9 & 65.3 & 163.4 & 58.9 & 58.9 & 60.1 & 61.1 \\
\hline \multirow[t]{2}{*}{ c880 } & 42 & avg & 669.3 & 393.4 & 36.6 & 160.7 & 768.6 & 134.7 & 69.4 & 75.5 & 46.8 & 74.2 \\
\hline & & dev & 681.1 & 385.9 & 36.7 & 161.4 & 763.9 & 125.9 & 68.3 & 72.1 & 46.3 & 72.5 \\
\hline \multirow[t]{2}{*}{ c2670 } & 78 & avg & 29.9 & 9.2 & 10.5 & 128.1 & 7.5 & 62.8 & 9.7 & 5.5 & 18.3 & 117.6 \\
\hline & & dev & 28.5 & 9.3 & 10.0 & 129.0 & 7.2 & 60.8 & 9.0 & 5.0 & 18.2 & 110.7 \\
\hline \multirow{2}{*}{$\mathrm{cm} 151 \mathrm{a}$} & 12 & avg & 5.3 & 16.1 & 6.4 & 6.5 & 15.9 & 372.5 & 5.4 & 5.3 & 110.3 & 4.2 \\
\hline & & dev & 4.7 & 16.3 & 5.9 & 6.1 & 15.8 & 403.2 & 5.1 & 4.7 & 109.0 & 3.5 \\
\hline \multirow[t]{2}{*}{$\mathrm{cu}$} & 13 & avg & 22.5 & 16.1 & 48.5 & 80.2 & 162.6 & 253.3 & 182.1 & 30.7 & 225.8 & 11.1 \\
\hline & & dev & 22.2 & 15.9 & 47.6 & 85.0 & 162.1 & 248.6 & 190.1 & 31.2 & 246.0 & 10.2 \\
\hline \multirow[t]{2}{*}{$\operatorname{misex} 3$} & 14 & avg & 326.6 & 28.1 & 579.5 & 337.1 & 303.1 & 234.0 & 47.0 & 54.5 & 131.4 & 79.4 \\
\hline & & dev & 336.0 & 27.1 & 576.0 & 324.3 & 287.3 & 243.1 & 45.9 & 54.4 & 127.2 & 74.4 \\
\hline \multirow[t]{2}{*}{ frg 1} & 25 & avg & 39866.3 & 70.1 & 471.6 & 104225.8 & 1709.9 & 989.2 & 3025.0 & 12890.1 & 38287.1 & 1956.8 \\
\hline & & dev & 38715.4 & 70.9 & 456.9 & 104031.3 & 1740.4 & 1018.6 & 2954.8 & 12954.8 & 38180.4 & 1992.8 \\
\hline \multirow[t]{2}{*}{ too_large } & 36 & avg & 636.5 & 2559.8 & 104685.6 & 1169.4 & 640.6 & 1302.6 & 614.6 & 738.6 & 711.5 & 7888.4 \\
\hline & & dev & 616.7 & 2667.7 & 103407.8 & 1176.7 & 609.8 & 1272.8 & 611.5 & 731.0 & 658.5 & 7798.7 \\
\hline \multirow[t]{2}{*}{$\mathrm{t} 481$} & 16 & avg & 810.6 & 503.9 & 415.2 & 53.3 & 3.1 & 1164.9 & 23.0 & 383.2 & 646.1 & 427.1 \\
\hline & & dev & 760.4 & 508.1 & 425.4 & 52.0 & 2.6 & 1088.7 & 22.4 & 364.2 & 674.5 & 440.8 \\
\hline
\end{tabular}

terms of comparing just the number of simulations to required Haar coefficients, we see that each technique is approximately equal since of the 21 benchmark functions in Table VI, 13 required fewer coefficients than random simulations.

However, we must point out the very important fact that the computational overhead required to obtain a single Haar coefficient is not equal to that for performing a simulation. Furthermore, the assumption that the Haar coefficients are equally likely to occur also biases these results to some degree since the subset of coefficients resulting from a specific partial HSD will have mutual dependence due to the definition of the transform.
Nevertheless, we can conclude that the use of Haar coefficients does appear to yield as much information as random simulations over this sample of benchmark functions. The importance of this result is that schemes that allow for the computation of Haar coefficients more efficiently than a netlist simulation can be used to increase the effectiveness of statistical verification.

\section{CONCLUSION}

A method for probabilistically determining the equivalence of two Boolean functions has been developed and

TABLE VI Average number of Haar coefficients before a mismatch occurs

\begin{tabular}{|c|c|c|c|}
\hline Circuit & Inp & Avg number coefficients & Avg number simulations \\
\hline 9sym-hdl & 9 & 2.7 & 5.9 \\
\hline c2670.329 & 78 & 39.9 & 29.1 \\
\hline c432.432GAT & 36 & 81.1 & 43.2 \\
\hline c499.OD31 & 41 & 74.2 & 252.4 \\
\hline c880.880GAT & 42 & 242.9 & 119.1 \\
\hline cc. 10 & 7 & 16.0 & 3.8 \\
\hline $\mathrm{cm} 150 \mathrm{a}$ & 21 & 7373.9 & 55.1 \\
\hline cm151a.m & 12 & 54.8 & 22.1 \\
\hline cm162a.r & 11 & 33.5 & 27.3 \\
\hline$c u . v$ & 13 & 103.3 & 223.5 \\
\hline dalu.O7 & 57 & 3133.2 & 3584.3 \\
\hline frg $1 . d 0$ & 25 & 20349.2 & 26958.6 \\
\hline misex3.12 & 14 & 212.1 & 1586.0 \\
\hline $\operatorname{mux}$ & 21 & 29810.1 & 93.1 \\
\hline pcler8.q0 & 13 & 641.4 & 1567.7 \\
\hline pm1.c0 & 9 & 25.4 & 90.5 \\
\hline rd53-hdl.out $<2>$ & 5 & 6.8 & 9.8 \\
\hline$t 481$ & 16 & 443.0 & 2214.2 \\
\hline too_large.nO & 36 & 12094.8 & 94946.1 \\
\hline$x 2 . \bar{p}$ & 10 & 15.9 & 31.3 \\
\hline$z 4 m l .24$ & 7 & 7.4 & 32.1 \\
\hline
\end{tabular}


presented. We have combined the use of two notions; partial BDDs [16] and the computation of Haar spectral coefficients using a BDD as a HSD [9]. The probabilistic framework has been derived for the equivalence checking problem.

Experimental results indicate that this approach can be a viable alternative for equivalence checking of functions that are difficult to represent completely. The experiments also indicate that this approach can be better in terms of required computational resources as compared to a repeated simulation approach.

\section{Acknowledgements}

This work was supported in part by NSF grants CCR9633085, SBE-9815371 and DAAD grant 315/PPP/gü-ab.

\section{References}

[1] Bryant, R.E. (1986) "Graph-based algorithms for Boolean function manipulation", IEEE Transactions on Computers 35, 677-691.

[2] Bryant, R.E. (1991) "On the complexity of VLSI implementations and graph representations of Boolean functions with application to integer multiplication", IEEE Transactions on Computers 40, $205-213$

[3] Camurati, P., Prinetto, P. and Torino, P.di. (1988) "Formal verification of hardware correctness: introduction and survey of current research", IEEE Computer Magazine July, 8-19.

[4] Devadas, S. (1993) "Comparing two-level and ordered binary decision representations of logic functions", IEEE Transactions on CAD 12(5), 722-723.

[5] Devadas, S., Tony Ma, Hi-Keung and Newton, R. (1988) "On the verification of sequential machines at differing levels of abstraction", IEEE Transactions on Computers 7(6), 713-722.

[6] Drechsler, R. and Becker, B. (1998) Binary Decision DiagramsTheory and Implementation (Kluwer Academic Publishers, Dordrecht).

[7] Drechsler, R., Hett, A. and Becker, B. (1997) "Symbolic simulation using decision diagrams", Electronic Letters 33(8), 665-667.

[8] Falkowski, B.J. and Chang, C.-C. (1994) "Efficient algorithms for forward and inverse transformations between Haar spectrum and binary decision diagram", International Phoenix Conference on Computers and Communications, pp 497-503.

[9] Hansen, J.P. and Sekine, M. (1997) "Decision diagram based techniques for the Haar wavelet transform", International Conference on Information, Communication and Signal Processing, pp 59-63.

[10] Hurst, S.L. (1981) "The Haar transform digital network synthesis", International Symposium on Multi-Valued Logic, pp 10-18.

[11] Hurst, S.L., Miller, D.M. and Muzio, J.C. (1985) Spectral Techniques in Digital Logic (Academic Press Publishers, New York).

[12] Jain, J., Bitner, J., Fussell, D. and Abraham, J. (1992) "Probabilistic verification of Boolean functions", Formal Methods in System Design: An International Journal 1(1), 63-118.
[13] Keutzer, K. (1987) "Dagon: technology binding and local optimization by dag matching", Design Automation Conference, pp 341-347.

[14] Madre, J.C. and Billion, J.-P. (1988) "Proving circuit correctness using formal comparison between expected and extracted behaviour", Design Automation Conference, pp 205-209.

[15] Malik, S., Wang, A.R., Brayton, R.K. and Sangiovanni-Vincentelli, A.L. (1988) "Logic verification using binary decision diagrams in a logic synthesis environment", International Conference on CAD, pp 6-9.

[16] Ross, D.E., Butler, K.M., Kapur, R. and Mercer, M.R. (1991) "Fast functional evaluation of candidate OBDD variable ordering", European Conference on Design Automation, pp 4-9.

[17] Stavridou, V., Barringer, H. and Edwards, D.A. (1988) "Formal specification and verification of hardware: a comparative case study”, Design Automation Conference, pp 197-204.

[18] Thornton, M.A. (1997) "Modified Haar transform calculation using digital circuit output probabilities", International Conference on Information, Communication and Signal Processing, pp 52-58.

\section{Authors' Biographies}

Mitchell Thornton is an Associate Professor in the Electrical and Computer Engineering Department at Mississippi State University. He has served on the faculty at the University of Arkansas for four years and has five years of industrial experience. His research interests include systems synthesis and verification, computer architecture and arithmetic. He is a senior member of the IEEE and the IEEE Computer Society.

Rolf Drechsler received his diploma and Ph.D. degree in computer science from the J.W. Goethe-University in Frankfurt am Main, Germany, in 1992 and 1995, respectively. He was with the Institute of Computer Science at the Albert-Ludwigs-University of Freiburg in Breisgau, Germany and is now at the University of Bremen. He published two books with Kluwer Academic Publishers, one on BDD techniques co-authored by Bernd Becker and one on using evolutionary algorithms for VLSI CAD. His research interests include verification, logic synthesis, and evolutionary algorithms.

Wolfgang Günther received his diploma in computer science from the Albert-Ludwigs-University of Freiburg in Breisgau, Germany in 1998. He is currently working at the same institute as a $\mathrm{PhD}$ student in the group of Bernd Becker. His research interests include formal verification, logic synthesis, and evolutionary algorithms. 

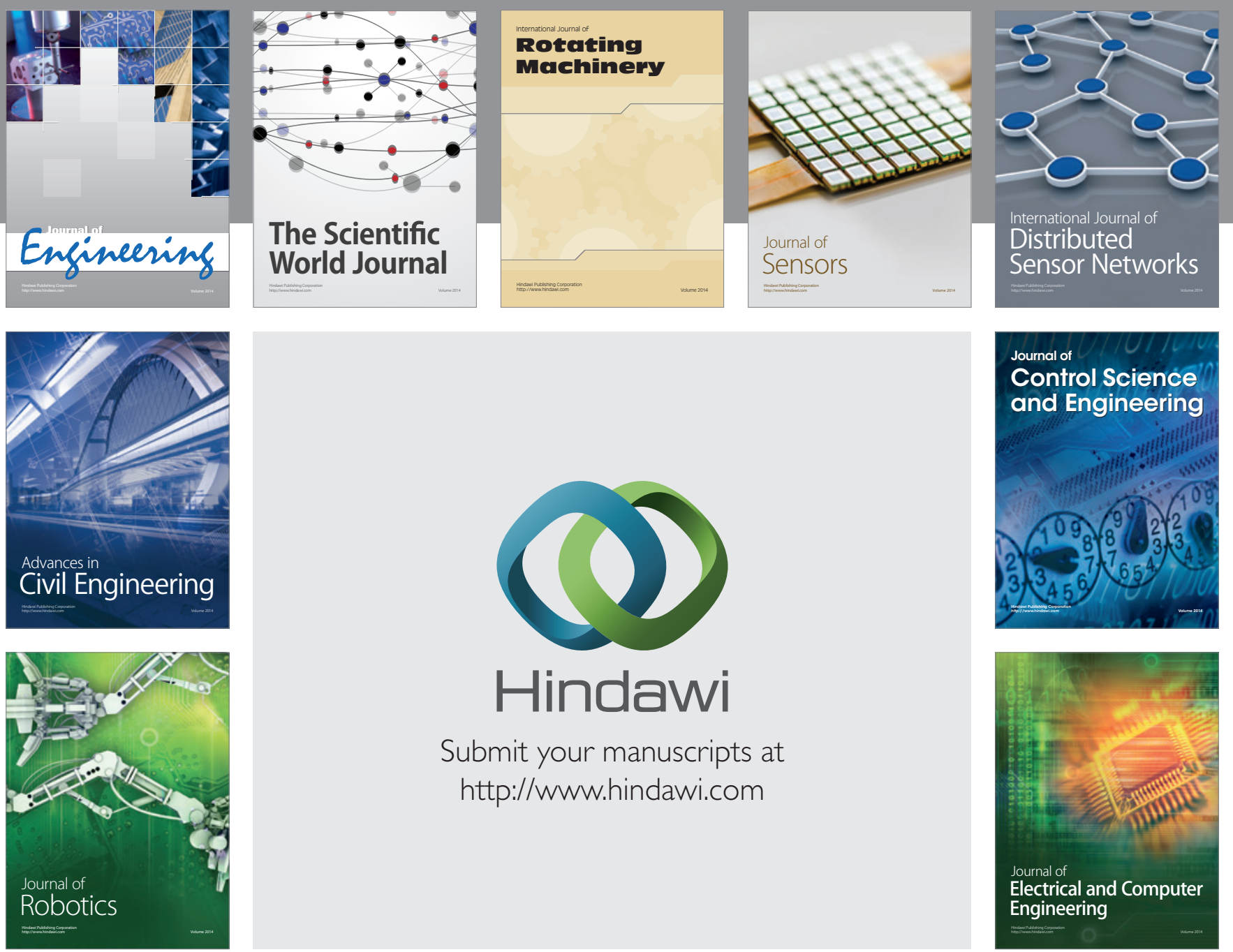

Submit your manuscripts at

http://www.hindawi.com
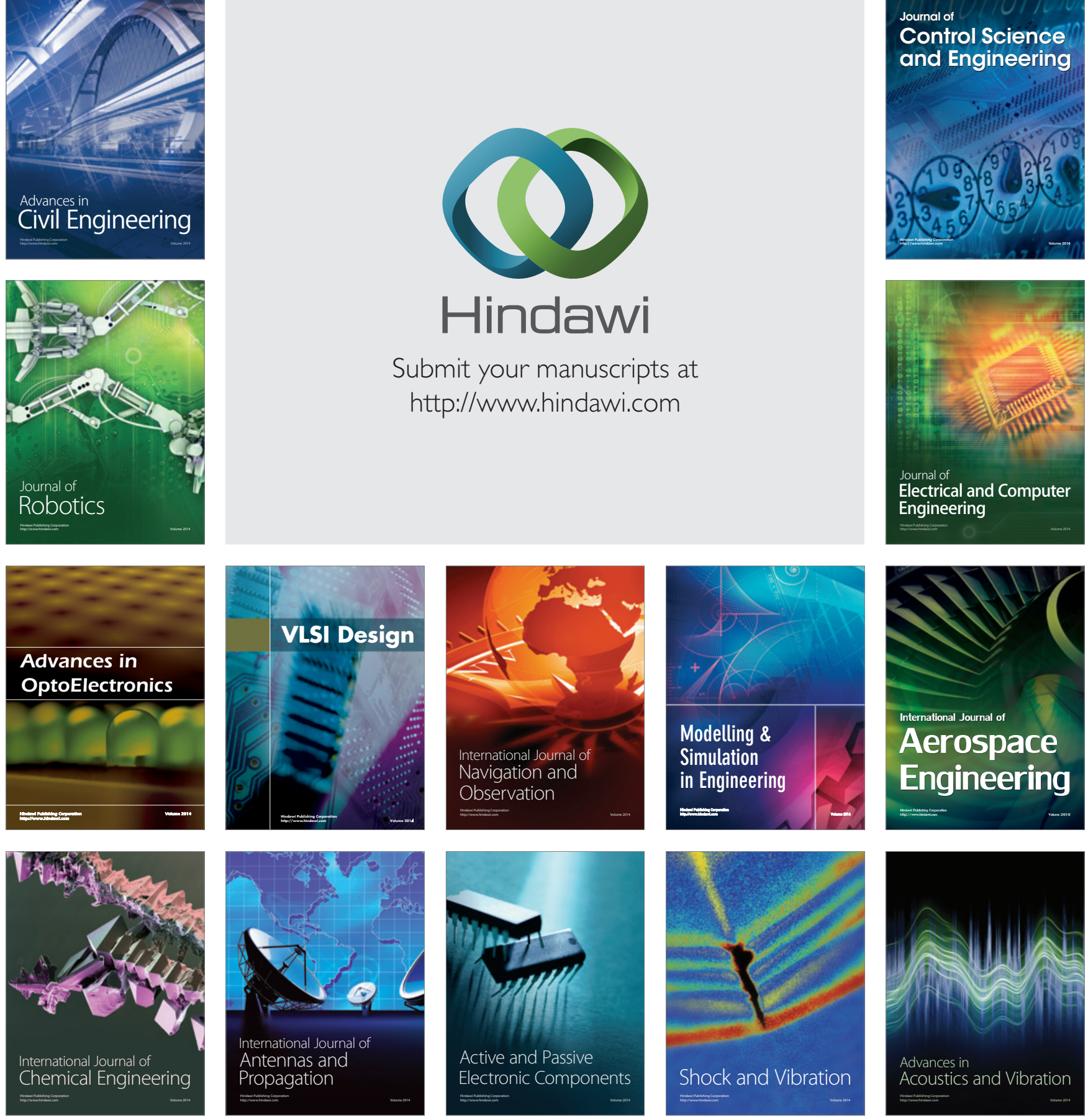